\title{
EXAMPLES OF HIGHER-DIMENSIONAL SLICE KNOTS WHICH ARE NOT RIBBON KNOTS
}

\author{
L. R. HITT ${ }^{1}$
}

\begin{abstract}
A definition for ribbon $n$-knot is given which reduces to the previously studied definitions for $n=1$ and $n=2$. It is shown that for each $n>2$ there is a slice $n$-knot which is not a ribbon $n$-knot.
\end{abstract}

1. Introduction. In $\left[\mathbf{F}_{2}\right]$, Fox presented a definition of ribbon knot for knots of $S^{1}$ in $S^{3}$, and posed the question, "Is every slice knot a ribbon knot?" This paper considers the higher dimensional analogue of this question.

Yajima [Yaj] has shown that Example 12 of [ $\left.\mathbf{F}_{\mathbf{1}}\right]$ is a slice 2-knot which is not a "simply knotted 2-sphere". When Yanagawa [Yan] showed that the definition of simply knotted 2-sphere was equivalent to his definition of ribbon 2-knot, he pointed out that the Fox example was thus a slice 2-knot which is not a ribbon 2-knot. Using different procedures, we construct examples here of slice $n$-knots which are not ribbon $n$-knots, for $n \geqslant 2$ (the classical case, $n=1$, remains unsettled).

In $\S 2$, the definition of ribbon $n$-knot is given (see $\left[\mathbf{H}_{1}\right],\left[\mathbf{H}_{2}\right],[\mathbf{R}]$ for a general discussion of ribbon $n$-knots) and a technical lemma is presented. This lemma is used in $\$ 3$ to single out algebraic conditions on an $n$-knot $(n \geqslant 2)$ complement which are sufficient to imply that the knot is nonribbon. Examples of knots which are slice, and which satisfy these algebraic conditions (and hence are nonribbon) are presented in $\$ 4$.

ACKNOWLEDGEMENTS. The results in this paper form a portion of the author's doctoral dissertation directed by Professor D. W. Sumners at Florida State University. His guidance in obtaining these results is very gratefully acknowledged.

2. Definitions and preliminary results. The definitions are given here in the smooth category, although they, and the results which follow, can be easily adjusted for the locally flat PL case. Let $n \geqslant 1$. An $n-k n o t, K=\left(S^{n+2}, f S^{n}\right)$, is a smooth oriented $(n+2, n)$-sphere pair, i.e., $f S^{n}$ is a smooth submanifold of $S^{n+2}$ and $f$ is a homeomorphism of $S^{n}$ onto the submanifold. The exterior of an $n-k n o t\left(S^{n+2}, f S^{n}\right)$ is the compact manifold with boundary obtained by removing an open tubular neighborhood of $f S^{n}$ from $S^{n+2}$. Note that by

\footnotetext{
Presented to the Society, April 7, 1978; received by the editors July 25, 1978.

AMS (MOS) subject classifications (1970). Primary 57C45.

Key words and phrases. Ribbon knot, slice knot, infinite cyclic covering space, knot cobordism.

${ }^{1}$ Research partially supported by a grant from the University of South Alabama Research Committee.
} 
Alexander duality, the exterior of a knot is a homology $S^{1}$.

A ribbon $n$-knot $K=\left(S^{n+2}, f S^{n}\right)$ is an $n$-knot such that $f S^{n}$ bounds an immersed $(n+1)$-disk in $S^{n+2}$, all the singularities of which are of the type depicted in Figure 1. More precisely, there must be an immersion $h: D^{n+I} \rightarrow$ $S^{n+2}$ such that $\left.h\right|_{\partial D^{n+1}}=f$, and such that the singular set of $h$ is a finite disjoint union of $n$-disks

$$
\bigcup\left\{A_{i}, B_{i} \mid 1 \leqslant i \leqslant m\right\} \subset D^{n+1},
$$

where for each $i, A_{i} \subset D^{n+1}$ is proper, $B_{i} \subset \operatorname{int}\left(D^{n+1}\right), h\left(A_{i}\right)=h\left(B_{i}\right)$, and $h^{-1}\left(h\left(A_{i}\right)\right)=A_{i} \cup B_{i}$. This definition is valid for any $n>1$, and agrees with the previously studied definitions of Fox [ $F_{2}$ ] and Yanagawa [Yan] for $n=1$ and $n=2$, respectively.

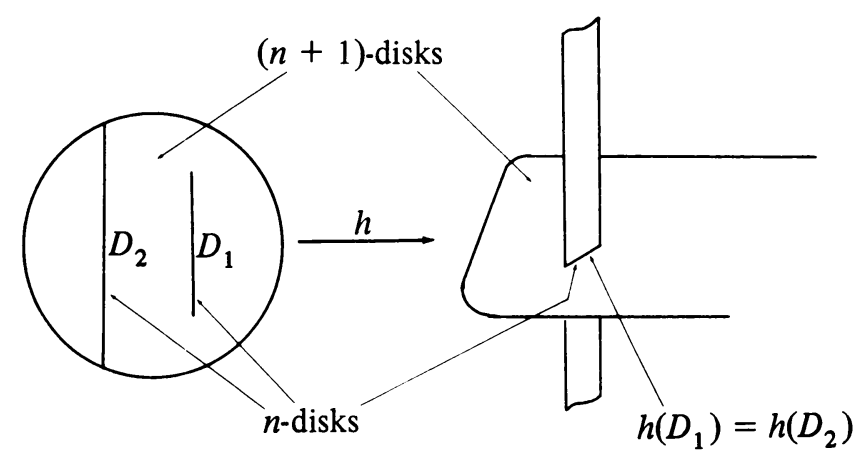

FIGURE 1

The lemma which follows will be applied to knot cobordism exteriors (\$3). The lemma itself, however, applies in a more general setting, which is developed here.

Let $X$ be a finite CW complex of dimension $n+1$, and suppose there is an epimorphism $\phi: \pi_{1}(X) \rightarrow \mathbf{Z}$. Let $\tilde{X}$ denote the covering space of $X$ which corresponds to $\operatorname{ker} \phi$, and let $t$ be a generator of the infinite cyclic group of covering transformations.

Following Milnor $[\mathbf{M}]$ and Levine $[\mathbf{L}]$, the rational chain groups $C_{q}(\tilde{X} ; \mathbf{Q})$ are finitely generated over the ring $\Gamma=\mathrm{Q}\left[t, t^{-1}\right]$ (the rational group ring of the group of covering transformations), the generators being in one-to-one correspondence with the $q$-cells of $X$. Since $\Gamma$ is Noetherian, the homology $H_{*}(\tilde{X} ; \mathbf{Q})$ is a finitely generated $\Gamma$-module. But in fact, $\Gamma$ is a principal ideal domain, so the theory of finitely generated modules over a principal ideal domain can be employed to aid in the analysis.

As in Milnor [M], the short exact sequence

$$
0 \rightarrow C_{*}(\tilde{X} ; \mathbf{Q}) \stackrel{t-1}{\rightarrow} C_{*}(\tilde{X} ; \mathbf{Q}) \rightarrow C_{*}(X ; \mathbf{Q}) \rightarrow 0
$$


(where the map $t-1$ is $\Gamma$-module multiplication) gives rise to a long exact sequence of homology:

$$
0 \rightarrow H_{n+1}(\tilde{X} ; \mathbf{Q}) \stackrel{(t-1) *}{\rightarrow} H_{n+1}(\tilde{X} ; \mathbf{Q}) \rightarrow H_{n+1}(X ; \mathbf{Q}) \stackrel{\partial_{*}}{\rightarrow} H_{n}(\tilde{X} ; \mathbf{Q}) \rightarrow \cdots
$$

Using a generalization of an argument due to Sumners $\left[\mathbf{S}_{\mathbf{1}}\right]$, we have the following:

LEMMA 1. Let $X, \tilde{X}$ be as above.

(i) If $H_{n+1}(X ; \mathbf{Q})=0$ then $H_{n+1}(\tilde{X} ; \mathbf{Z})=0$; also,

(ii) if $H_{n+1}(\tilde{X} ; \mathbf{Z})=0$ and if $(t-1)_{*}: H_{n}(\tilde{X} ; \mathbf{Q}) \rightarrow H_{n}(\tilde{X} ; \mathbf{Q})$ is an epimorphism, then $H_{n}(\tilde{X} ; \mathbf{Z})$ is $\mathbf{Z}$-torsion free.

Proof. (i) By assumption, $H_{n+1}(X ; \mathbf{Q})=0$, so $t-1: H_{n+1}(\tilde{X} ; \mathbf{Q}) \rightarrow$ $H_{n+1}(\tilde{X} ; \mathbf{Q})$ is an epimorphism (in fact, an isomorphism). Thus, $t-1$ divides every element in $H_{n+1}(\tilde{X} ; \mathbf{Q})$, which shows $H_{n+1}(\tilde{X} ; \mathbf{Q})$ is a torsion $\Gamma$-module.

On the other hand, since $C_{n+2}(\tilde{X} ; \mathbf{Q})=0, H_{n+1}(\tilde{X} ; \mathbf{Q})=Z_{n+1}(\tilde{X} ; \mathbf{Q})$ (the module of $n$-cycles). And since $\Gamma$ is a PID, $Z_{n+1}(\tilde{X} ; \mathbf{Q})$ is a free $\Gamma$-module. Thus, $H_{n+1}(\tilde{X} ; \mathbf{Q})=0$, since it is both torsion and free.

Applying the Universal Coefficient Theorem to $H_{n+1}(\tilde{X} ; \mathbf{Q})$, we see that $0=H_{n+1}(\tilde{X} ; \mathbf{Z}) \otimes_{\mathbf{Z}} \mathbf{Q}$. Thus, $H_{n+1}(\tilde{X} ; \mathbf{Z})$ is a torsion $\mathbf{Z}$-module. But, as before, $H_{n+1}(\tilde{X} ; \mathbf{Z})$ is also a free $\mathbf{Z}$-module. Thus, $H_{n+1}(\tilde{X} ; \mathbf{Z})=0$, as desired.

(ii) Let $\Lambda=\mathbf{Z}\left[t, t^{-1}\right]$, the integral group ring of the covering transformation group, and let $T_{n}$ denote the $\Lambda$-submodule of $H_{n}(\tilde{X} ; \mathbf{Z})$ consisting of all Z-torsion elements.

By Lemma II.8 in Kervaire [K], we can conclude that $T_{n-1}$ is a finite $\Lambda$-module from the assumption that

$$
(t-1)_{*}: H_{n}(\tilde{X} ; \mathbf{Q}) \rightarrow H_{n}(\tilde{X} ; \mathbf{Q})
$$

is an epimorphism. Now, multiplication induces an automorphism on $T_{n}$, so there is some positive integer $p$ such that $t^{p}$ induces the identity automorphism.

To exploit this observation, let $\tilde{X}_{p}$ denote the $p$-fold cyclic covering of $\tilde{X}$. From Shinohara and Sumners [SS], we have the following long exact sequence which relates the homology of $\tilde{X}_{p}$ to that of $\tilde{X}$ :

$$
\cdots \rightarrow H_{n+1}(\tilde{X} ; \mathbf{Z}) \rightarrow H_{n+1}(\tilde{X} ; \mathbf{Z}) \stackrel{\partial}{\rightarrow} H_{n}(\tilde{X} ; \mathbf{Z}) \stackrel{\left(t^{p}-1\right)}{\rightarrow} H_{n-1}(\tilde{X} ; \mathbf{Z}) \rightarrow \cdots
$$

Now, $T_{n} \subseteq \operatorname{ker}\left(\left(t^{p}-1\right)_{*}\right)=\operatorname{im} \partial$. But $H_{n+1}(\tilde{X} ; Z)=0$ by assumption, so $H_{n+1}\left(\tilde{X}_{p} ; \mathbf{Z}\right) \simeq{ }^{\partial}$ im $\partial$. Thus $T_{n}$ is embedded as a submodule of $H_{n+1}\left(\tilde{X}_{p} ; \mathbf{Z}\right)$. However, since $\tilde{X}_{p}$ has dimension $n+1, H_{n+1}\left(\tilde{X}_{p} ; \mathbf{Z}\right)$ is a free Z-module. Thus, $T_{n}=0$, i.e., $H_{n}(\tilde{X} ; \mathbf{Z})$ is $\mathbf{Z}$-torsion free.

Corollary 2. If $X$ is a homology $S^{1}$ which is homotopy equivalent to a finite $C W$ complex of dimension $n+1(n>1)$, then $H_{n}(\tilde{X} ; \mathbf{Z})$ is Z-torsion free. 
Proof. Let $n \geqslant 1$. Since $X$ is a homology $S^{1}$, it follows from the long exact sequence (1) that the homomorphism

$$
(t-1)_{*}: H_{n}(\tilde{X} ; \mathbf{Q}) \rightarrow H_{n}(\tilde{X} ; \mathbf{Q})
$$

is an epimorphism. The conclusion then follows from the lemma.

REMARK. Let $\left(S^{n+2}, f S^{n}\right)$ be an $n$-knot $(n>1)$, and let $X$ denote its exterior. Since $X$ is an $(n+2)$-manifold with nonempty boundary, it can be collapsed away from its boundary onto a finite $(n+1)$-complex. By the lemma, then, $H_{n}(\tilde{X} ; \mathbf{Z})$ is $\mathbf{Z}$-torsion free.

3. Handle decompositions of null-cobordisms. Two $n$-knots, $\left(S^{n+2}, f_{1} S^{n}\right)$ and $\left(S^{n+2}, f_{2} S^{n}\right)$, are cobordant if there is a smooth oriented proper submanifold $w$ of $S^{n+2} \times I$, with

$$
\partial w=\left(f_{1} S^{n} \times 0\right) \cup\left(-f_{2} S^{n} \times 1\right)
$$

where $w$ is homeomorphic to $S^{n} \times I$ and where $-f_{2} S^{n}$ denotes the embedding obtained from $f_{2}: S^{n} \rightarrow S^{n+2}$ by reversing the orientations on both $S^{n}$ and $S^{n+2}$. The pair $\left(S^{n+2} \times I, w\right)$ is called a knot cobordism. A knot is null-cobordant, or slice, if it is cobordant to the unknot. By the exterior of a knot cobordism $\left(S^{n+2} \times I, w\right)$, we mean the manifold $W=S^{n+2} \times I-\grave{\nu}(w)$ where $i(w)$ is an open tubular neighborhood of $w$ in $S^{n+2} \times I$. Note that knot cobordism exteriors are homology 1-spheres. Also, the exterior of a knot cobordism is a cobordism with boundary (in the usual, absolute, sense) between the exteriors of the two cobordant knots. As such, $W$ has a handle decomposition built up from either of the knot exteriors.

The following theorem will be used in $\$ 4$ to show that certain slice knots are nonribbon.

TheOREM 3. Let $\tilde{X}$ denote the infinite cyclic covering of the slice knot $K$. If, for some $q, H_{q}(\tilde{X} ; \mathbf{Z})$ has $\mathbf{Z}$-torsion, then any handle decomposition of any null-cobordism of $K$ built from the unknotted end has either $a(q+2)$-handle or an $(n-q+2)$-handle.

Proof. We will prove the contrapositive of the theorem. Let $W, X$, and $U$ denote the exteriors of the cobordism, the knot $K$, and the unknot, respectively. Suppose the negation of the conclusion, i.e., that there is some handle decomposition of $W$ of the form

$$
W \cong U \times I \cup\left\{h_{j}^{i} \mid 1 \leqslant i \leqslant n+2, i \neq q+2, i \neq n-q+2, j \in A_{i}\right\}
$$

where $h_{j}^{i}$ denotes a handle of index $i$ and $A_{i}$ is a finite indexing set.

Since handles are simply connected, they lift to any covering space to induce a handle structure, so

$$
\tilde{W} \cong U \times I \cup\left\{h_{\alpha}^{i} \mid i \neq q+2, i \neq n-q+2, \alpha \in B_{i}\right\}
$$

where the $B_{i}$ are infinite indexing sets. Let

$$
W_{q+1} \cong U \times I \cup\left\{h_{j}^{i} \mid i \leqslant q+1, j \in A_{i}\right\}
$$


and

$$
\tilde{W}_{q+1} \cong \tilde{U} \times I \cup\left\{h_{\alpha}^{i} \mid i \leqslant q+1, \alpha \in B_{i}\right\},
$$

where the diffeomorphisms are the ones from (2) and (3), respectively. The contrapositive will be proven, first by applying Corollary 2 to $W_{q+1}$ and using the assumption of no $(q+2)$-handle to show that $H_{q}(\tilde{W} ; \mathbf{Z})$ is torsion free, and then by using the assumption of no $(n-q+2)$-handle to conclude $H_{q}(\tilde{X})$ is torsion free.

To apply the corollary, note that $W_{q+1}$ is homotopy equivalent to a finite $(q+1)$-dimensional $\mathrm{CW}$ complex. Furthermore, since $W$ is obtained from $W_{q+1}$ by attaching handles of index greater than $q+2, H_{m}(W ; \mathbf{Z}) \simeq$ $H_{m}\left(W_{q+1} ; Z\right)$ for $m \leqslant q+1$. But $W$ is a homology $S^{1}$, so $H_{*}\left(S^{1} ; R\right) \cong$ $H_{*}\left(W_{q+1} ; R\right)$ for $R=\mathbf{Z}$, and by the Universal Coefficient Theorem, for $R$ arbitrary. By Corollary 2 , then, $H_{q}\left(\tilde{W}_{q+1} ; \mathbf{Z}\right)$ is Z-torsion free, and hence, so is $H_{q}(\tilde{W} ; \mathbf{Z})$.

Consider now the dual handle decomposition of $\tilde{W}$

$$
\tilde{W} \cong \tilde{X} \times I \cup\left\{\stackrel{*}{h}^{i} \mid i \neq q+1, i \neq n-q+1\right\},
$$

where $\stackrel{*}{h}_{\alpha}^{i}$ is the dual handle of $h_{\alpha}^{n+3-i}$. Since $\tilde{W}$ is obtained from $\tilde{X} \times I$ by attaching handles of index other than $q+1, H_{q+1}(\tilde{W}, \tilde{X} \times I ; Z)=0$. The long exact sequence of the pair $(\tilde{W}, \tilde{X} \times I)$ then shows that the inclusion induced homomorphism $i_{*}: H_{q}(\tilde{X} \times I ; \mathbf{Z}) \rightarrow H_{q}(\tilde{W} ; \mathbf{Z})$ is a monomorphism. Since $H_{q}(\tilde{W} ; \mathbf{Z})$ is torsion free, then so is $H_{q}(\tilde{X} ; \mathbf{Z})$, as desired.

4. Applications. We will make use of the following fact about ribbon knots.

THEOREM 4 (HITt [ $\left.\left.\mathbf{H}_{1}\right],\left[\mathbf{H}_{2}\right]\right)$. If $K$ is a ribbon $n$-knot $(n>1)$, then $K$ bounds a null-cobordism having a handle decomposition consisting entirely of 1 - and 2-handles from the unknotted end.

Combining the above theorem with Theorem 3, we have

THEOREM 5. If $\tilde{X}$ denotes the infinite cyclic covering of the exterior of an $n$-knot, $K$, for which $H_{q}(\tilde{X} ; \mathrm{Z})$ has $\mathrm{Z}$-torsion for some $q$ in the range $1<q<n$ -1 , then $K$ is not a ribbon knot.

REMARKs. (1) The theorem is meaningful only if $n>2$.

(2) In case $n=2$, any 2-knot $K$ for which $H_{1}(\tilde{X} ; \mathbf{Z})$ has Z-torsion is a slice knot (Kervaire [K]) which is not a ribbon knot. If we take $K$ to be Fox's Example 12 , since $H_{1}(\tilde{X} ; \mathbf{Z}) \cong \mathbf{Z} /(3)$, then $K$ is a slice 2 -knot which is not a ribbon 2-knot. Thus, Yanagawa's result is obtained as a special case of the theorem.

(3) Other slice 2-knots which are nonribbon can be obtained by twist-spinning (see [Z]) certain classical knots. For example, let $K$ be any 2-bridge knot. Schubert [Sc, Satz 6] has shown that the 2-fold branched cover of any 2-bridge knot, $K$, is a lens space, say $L(p, q)$. Then by Zeeman's fibration 
theorem $[\mathrm{Z}], H_{1}(\tilde{Y}) \cong H_{1}(L(p, q)) \cong \mathbf{Z} /(p)$, where $Y$ denotes the exterior of the 2-twist-spin of $K$.

(4) Similarly, by calculating the homology of the $k$-fold branched covers of the trefoil, one sees that for the $k$-twist-spin of the trefoil

$$
H_{1}(\tilde{Y}) \cong\left\{\begin{array}{l}
\mathbf{Z} /(3) \quad \text { if } k \equiv \pm 2(\bmod 6) \\
\mathbf{Z} /(2) \oplus \mathbf{Z} /(2) \quad \text { if } k \equiv 3(\bmod 6)
\end{array}\right.
$$

To obtain higher dimensional examples of slice knots which are not ribbon knots, we will $p$-spin the 2 -knot examples. The $p$-spin of an $n$-knot $K(p>0$, $n>1)$ is defined as $\sigma_{p}(K)=\partial\left(\bar{K} \times D^{p+1}\right)$ where $\bar{K}$ is the disk pair associated with $K$ obtained by removing the interior of an unknotted disk pair from $K$. Note that $\sigma_{p}(K)$ is a slice $(n+p)$-knot.

In $\left[\mathbf{S}_{2}\right.$ ], Sumners discusses the relation between the algebraic invariants of a knot and its $p$-spin, proving

Theorem 6 (Sumners). Let $X$ denote the exterior of a knot $K$, and $Y$ the exterior of the p-spin of $K$. Then

$$
H_{i}(\tilde{Y}) \cong_{\Lambda}\left\{\begin{array}{l}
H_{i}(\tilde{X}) \quad \text { if } i \leqslant p \\
H_{i}(\tilde{X}) \oplus H_{i-p}(\tilde{X}) \quad \text { if } i>p
\end{array}\right.
$$

Using Sumners' theorem, we have the following

THEOREM 6. Let $K$ be an $n$-knot for which $H_{q}(\tilde{X} ; \mathbf{Z})$ has $\mathbf{Z}$-torsion for some $q$ in the range $1<q<n-1$. Then for any $p>0, \sigma_{p}(K)$ is a slice knot which is not a ribbon knot.

Proof. Let $Y$ denote the exterior of $\sigma_{p}(K)$. By Theorem $6, H_{q}(\tilde{Y})$ contains $H_{q}(\tilde{X})$ as a $\Lambda$-submodule. Thus, $H_{q}(\tilde{Y})$ has $Z$-torsion, and $\sigma_{p}(K)$ is nonribbon by Theorem 5 .

COROLlARY 7. For each $n \geqslant 2$, there is a slice $n$-knot which is not a ribbon $n-k n o t$.

\section{REFERENCES}

[F, R. H. Fox, A quick trip through knot theory, Topology of 3-Manifolds and Related Topics, Prentice-Hall, Englewood Cliffs, N. J., 1961, pp. 120-167.

$\left[\mathbf{F}_{2}\right]$, Some problems in knot theory, Topology of 3-Manifolds and Related Topics, Prentice-Hall, Englewood Cliffs, N. J., pp. 168-176.

[H. $\left.\mathrm{H}_{1}\right]$ L. R. Hitt, Handlebody presentations of knot cobordisms, $\mathrm{Ph}$. D. Dissertation, Florida State University, 1977.

$\left[\mathrm{H}_{2}\right] \ldots$, Ribbon $n$-knots, preprint, University of South Alabama.

[K] M. A. Kervaire, Les noeuds de dimensions supérieures, Bull. Soc. Math. France 93 (1965), 225-271.

[L] J. Levine, Polynomial invariants of knots of codimension two, Ann. of Math. (2) 84 (1966), 537-554.

[M] J. Milnor, Infinite cyclic coverings, Conference on the Topology of Manifolds, Prindle, Weber \& Schmidt, Boston, Mass., 1968, pp. 115-133.

[R] D. Roseman, Ribbon knots, preprint, University of Iowa.

[Sc] H. Schubert, Knoten mit zwei Brücken, Math. Z. 65 (1950), 133-170. 
[SS] Y. Shinohara and D. W. Sumners, Homology invariants of cyclic coverings with applications to links, Trans. Amer. Math. Soc. 163 (1972), 101-121.

[S $]$ D. W. Sumners, $\mathrm{H}_{2}$ of the commutator subgroup of a knot group, Proc. Amer. Math. Soc. 28 (1971), 319-320.

$\left[\mathbf{S}_{2}\right]$ State University.

[Yaj] T. Yajima, On simply knotted spheres in $R^{4}$, Osaka J. Math. 1 (1964), 133-152.

[Yan] T. Yanagawa, On ribbon 2-knots, the 3-manifold bounded by the 2-knots, Osaka J. Math. 6 (1969), 447-464.

[Z] E. C. Zeeman, Twisting spun knots, Trans. Amer. Math. Soc. 115 (1965), 471-495.

Department of Mathematics, University of South Alabama, Mobile, Alabama 36688 